\title{
Optimal hoist scheduling of a deep level mine twin rock winder system for demand side management
}

\author{
Werner Badenhorst*, Jiangfeng Zhang, Xiaohua Xia \\ Centre of New Energy Systems, Department of Electrical, Electronic and Computer Engineering, University of Pretoria, Pretora 0002, South Africa
}

\section{A R T I C L E I N F O}

\section{Article history:}

Received 4 June 2010

Received in revised form

23 November 2010

Accepted 16 December 2010

Available online $\mathrm{xxx}$

\section{Keywords:}

Load scheduling

Demand side management

Mixed integer linear programming

Model predictive control

\begin{abstract}
A B S T R A C T
This paper presents a near optimal hoist scheduling and control program for rock winders found in South African deep level mines in the context of demand side management and time-of-use (TOU) tariffs. The objective is to achieve a set hoist target at minimum energy cost within various system constraints. The development of a discrete dynamic and constrained mixed integer linear programming model for a twin rock winder system is presented on which a half-hourly model predictive control (MPC) algorithm containing an adapted branch and bound methodology is applied for near optimal scheduling. Simulation results illustrate the effectiveness of the control program by minimising the energy costs through scheduling according to the TOU tariff and controlling output and ore levels within their boundaries even in the case of significant random delays in the system. Scheduling according to the TOU tariff shows a possible $30.8 \%$ reduction in energy cost while approximately $6 \mathrm{~h}$ of delays in the system resulted in a mere $14 \%$ increase in energy cost.
\end{abstract}

(C) 2010 Elsevier B.V. All rights reserved.

\section{Introduction}

In order to meet the projected global electricity demand increase of $45 \%$ between 2006 and 2030, ${ }^{1}$ supply capacity needs to be increased and the rate of demand increase needs to be reduced through the effective demand side management (DSM) of the electricity market. The need for additional future generation capacity can however be reduced through a reduction of demand [1,2]. In light of the aforementioned Africa's largest electricity supplier, Eskom, has made DSM a priority with respect to the efficient use of electricity and reducing peak demand by $3000 \mathrm{MW}$ between April 2007 and April 2011 and a further 5000 MW by March 2026. ${ }^{2}$

Industry in South Africa consumes approximately $65 \%$ of all electrical energy of which the mining sector is the largest representing approximately $24 \%$ of industry consumption and $16 \%$ of total electrical energy consumption in South Africa. ${ }^{3}$ DSM projects that have successfully been implemented in deep level gold mines are primarily on the underground pumping, cooling and lighting systems [3]. Another potential system identified for DSM in deep level mines

\footnotetext{
* Corresponding author. Tel.: +27 12420 2587; fax: +27 123625000.

E-mail address: werner.badenhorst@eng.up.ac.za (W. Badenhorst).

1 IEA, IEA World Energy Outlook 2008 Executive Summary, <http://www. worldenergyoutlook.org>.

2 Eskom, Eskom Energy Efficiency and Demand Side Management Programme Overview, 2008.

${ }^{3}$ DME, Department Minerals and Energy, Republic of South Africa, Digest of South African Energy Statistics, 2006.
}

is the rock winders, which are responsible for hoisting ore and rock from underground to the surface where the gold is then extracted.

The primary objective of the problem presented in this paper is the development of a near optimal half hourly hoist control scheduling program for a deep level mine twin rock winder system in order to achieve a set hoist target at minimum energy cost based on a time-of-use (TOU) tariff while operating within various physical and operational constraints.

The paper first presents a brief summary in Section 2 of relevant literature applied in achieving the primary objective. Section 3 formulates the problem through the development of a physically based, discrete dynamic and constrained mixed integer linear programming (MILP) model of the rock winder system. Section 4 presents a model predictive control (MPC) framework with a receding horizon method ensuring that future scheduled hoists are updated in the event of delays and other system changes affecting system constraints and conditions. Section 5 describes the closed loop MPC algorithm in which measured system states and delays are used as feedback every $30 \mathrm{~min}$. The algorithm also incorporates an adapted branch and bound methodology to obtain a near optimal mixed integer hoist schedule solution. Finally in Section 6 the program simulates and plots hoist schedules for the winders indicating the number of hoists and predicted system state levels for each half hour period over a set number of periods. The effectiveness of the MPC algorithm is illustrated by comparing the impact of using a TOU versus flat rate tariff as well as the inclusion delays into the winder system. The results presented focuses on the near optimality of the integer solution and the ability of the MPC algorithm 
to absorb the primary dynamic factor in the system, namely the uncertainty of duration and time of occurrence of system delays.

\section{Literature research}

The first significant contribution towards DSM in deep level mines was made in [4] in which an integrated electricity end-use planning methodology in deep level mines is proposed. It is shown in [4] that rock winders represent approximately $15 \%$ of the energy consumption on a deep level goldmine making this focussed study very relevant and applicable within large mining industry in South Africa.

The modelling of the rock winder system in this paper is primarily based on the physically based models such as presented in [5] for scheduling load for a flour mill to minimise electricity costs; providing an optimal load management strategy for an air conditioning plant utilising load shifting [6]; for peak-load management in steel plants [7] and for an optimal control model for load shifting of a colliery conveyer transport system [8].

Closed loop control is achieved through the application of MPC. MPC's ability to handle constraints and simple models along with its robustness and closed-loop stability has made MPC one of the most widely used multivariable control algorithms in many industry applications [9-11] including power systems. Amongst other MPC is applied in [12] for load shifting of a water pumping scheme using binary integer programming optimisation taking into account both TOU and maximum demand charges. Another example includes an MPC approach to the dynamic economic dispatch problem of generators with ramp rate constraints in [13]. A number of applications combine MPC with minimisation or optimisation problems of which a few include variable-air-volume boxes [14], fluidised furnace reactors [15], boiler start-ups [16] and in the design and operation of distributed energy resources [17].

In contrast to existing studies, the rock winder problem presented in this paper applies the above mentioned techniques in a mining environment with the added requirement of an integer solution to the optimised scheduling problem. The formulation of the rock winder problem into a constrained integer linear programming model along with a solution methodology using an adapted Branch and Bound methodology without feedback control was first introduced in [18]. The reformulation of the problem in [18] for application in a MPC algorithm has been presented in [19]. These two papers provide preliminary results for the complete and concise formulation of the study presented in this paper in which the adapted Branch and Bound methodology is incorporated within a MPC algorithm to obtain a near optimal hoist schedule for a twin rock winder system.

\section{Problem formulation}

\subsection{Nomenclature}

For better comprehension of the problem formulation to follow a nomenclature is provided first.

$C$ cost vector for model horizon $N$

$c_{k}$ energy cost per kWh during period $k$

$D$ the number of days within the control horizon $H$

$E_{x}$ energy consumption per hoist for winder $x$

$f f_{x}$ friction factor accounting for the friction load for winder $x$ such that $0 \leq f f<0.3$

$g$ gravitational acceleration as $9.81 \mathrm{~m} / \mathrm{s}^{2}$

$H$ the control horizon and the number of periods therein calculated as $H=48 D$ $h_{x}$ vertical winding depth or hoist height for winder $x$ in meters

$J$ energy cost and linear objective function

$M_{\text {blast }}$ one day's target production or ore bearing rock in tons to be blasted in the reefs

$M_{\text {min }}$ daily minimum required tons to be hoisted to surface

$m_{1}^{k}, m_{2}^{k}$ tons stored at the start of period $k$ in respectively the change-over and orepass system.

$m_{i n}^{k}$ feed in rate of tons of rock from the reefs into the orepass system during period $k$.

$N$ model horizon

$\eta_{x}$ winder efficiency of winder $x$ measured as the ratio of shaft output power required over total electrical input power required

$P$ prediction horizon

$R_{x}$ set skip payload per hoist for winder $x$ in tons

$T_{m x}$ number of half hourly periods to complete the maintenance or test period of winder $x$

$T_{S x}$ starting time at either top or bottom of the hour for the maintenance or test period of winder $x$

$u$ vector containing the number of scheduled hoists for all rock winders for each period in $H$

$u_{x}^{k}$ number of hoists for winder $x$ during period $k$.

\subsection{Ore transport and rock winder system overview}

The schematic layout in Fig. 1 is of a typical deep level ore transport system of which the most critical component is the rock winder. The blasted rock from the reefs is transported to the shaft area via orepasses, crushers and conveyor belts feeding flasks that weigh off a set payload to be loaded into the skips in which the rock is hoisted to surface by the rock winder. ${ }^{4}$ The ore is then stored on surface in stockpiles or silos awaiting transport to the gold plant where the gold is extracted from the ore-bearing rock.

The twin rock winder system used in this study consists of an underground and a surface winder as indicated in the diagram of Fig. 2. Each day's blasted rock is transported from the reefs and stored in the underground orepass system. From the orepass system the rock is conveyed into a flask where the rock is weighed to $R_{g}=13.5$ tons, before being emptied into the underground winder's skip. The loaded skip is then hoisted by the underground winder in the sub-shaft and emptied into a change-over. From the changeover the rock is conveyed into a flask where the rock is now weighed to $R_{S}=23.5$ tons, before being emptied into the surface winder's skip. The loaded skip is then hoisted to surface where it is in turn emptied onto a conveyance transporting the rock to a surface stockpile with a capacity that will for the purposes of this study be assumed to be infinite.

There are two primary uncertainties or dynamic factors within the rock winder system. The first and least significant is the feedin rate of ore from the reefs into the orepass system that differs from day to day. The feed-in rate can however be averaged over $1 \mathrm{~h}$ periods within a $24 \mathrm{~h}$ period based on historical records and the $24 \mathrm{~h}$ cyclic operation schedule of a mine with a high level of certainty. The second factor having by far the biggest impact on the operational hours of the rock winder system is that of unscheduled or unplanned system delays. This is because of the uncertainty in duration and time of occurrences of these delays which can occur at any time and averaged at 152 and 206 min per day for the surface and underground winder respectively during this study. If the MPC algorithm can therefore absorb the effects of such delays, the

\footnotetext{
${ }^{4}$ AngloGold Ashanti, AngloGold Ashanti Virtual Mine Tour, <http://www anglogold.co.za>.
} 


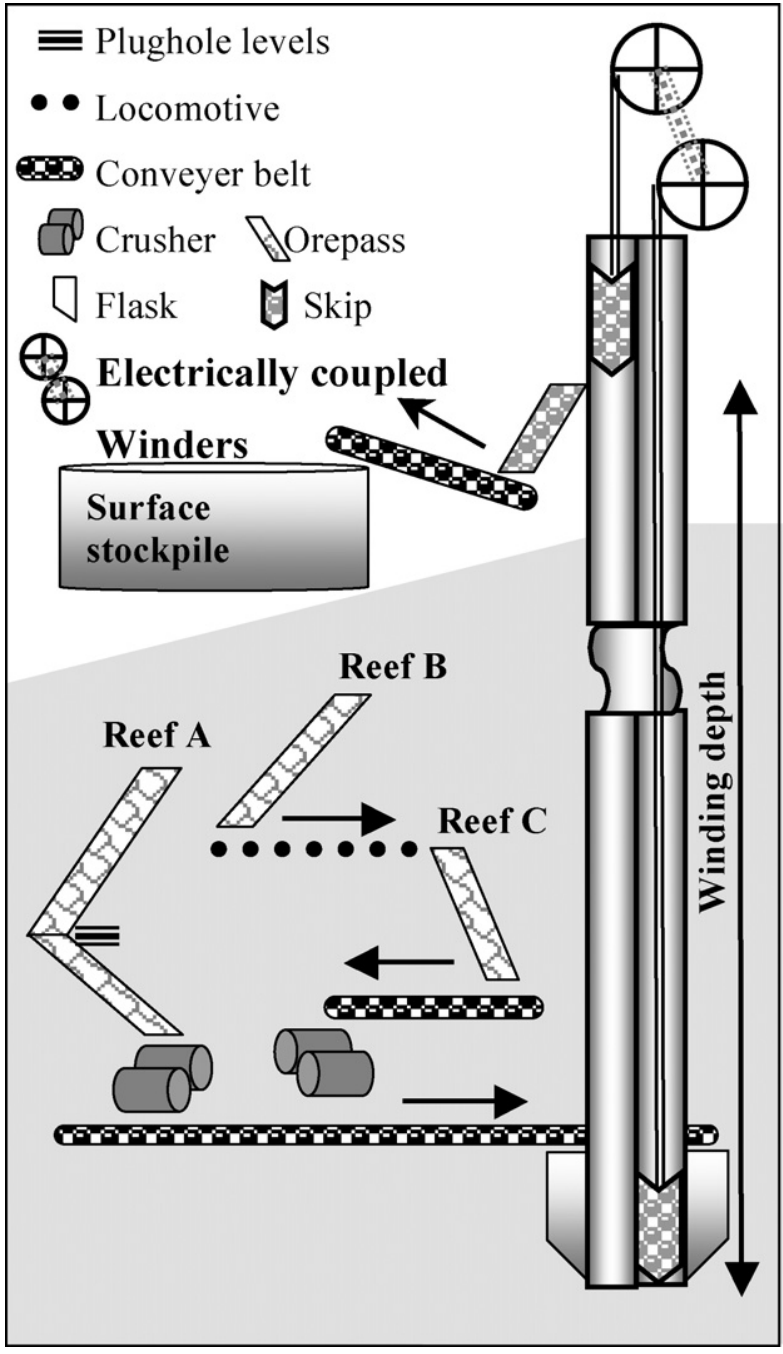

Fig. 1. Schematic layout of a typical deep level ore transport system.

algorithm will easily be able to absorb other minor disturbances within the system.

\subsection{Objective function}

As the primary objective is to minimise the energy cost of the winders, the objective function will take the form of an energy cost function similar to that developed in [5-8]. The first part of the objective function entails the energy consumption per hoist calculated using Eq. (1):

$E_{x}=\frac{\left(1+f f_{x}\right) R_{x} \times g \times h_{x}}{\eta_{x} \times 3600}[\mathrm{kWh}]$

The payload, hoist height and efficiency of each winder can safely be assumed to be constant meaning that the energy consumption per hoist can also be assumed constant. Therefore the energy consumption of a winder during period $k$ can be calculated as the product of the number of hoists during that period and the energy consumption per hoist. For the surface winder this energy consumption during period $k$ can be written as $u_{s}^{k} E_{s}$ and for the underground winder $u_{\mathrm{g}}^{k} E_{g}$.

In accounting for the cost component of the objective function it was noted that the mine operated on a time-of-use (TOU) tariff

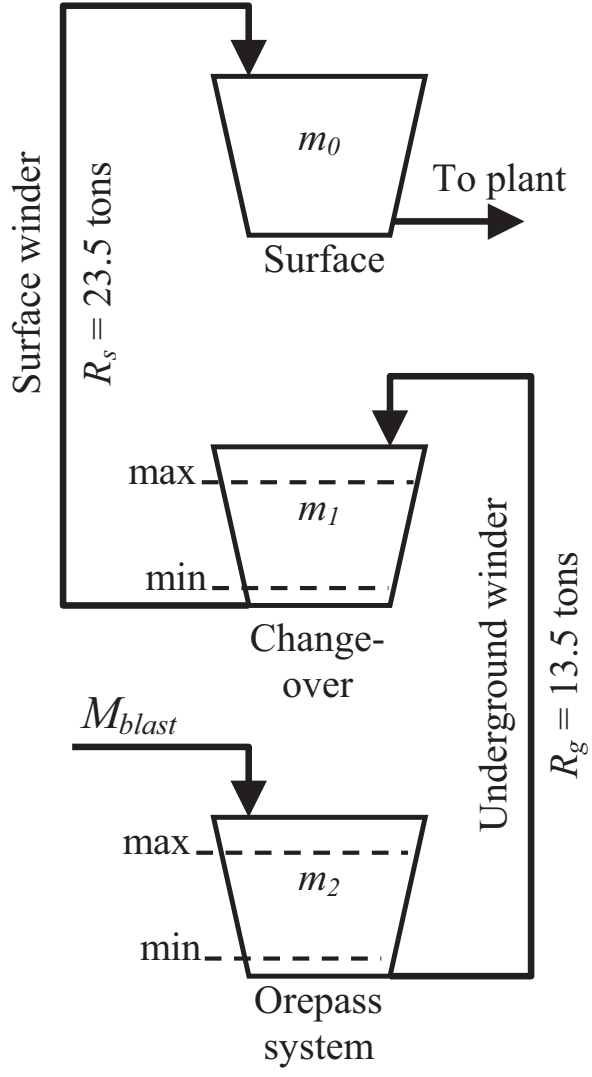

Fig. 2. Process flow diagram of a twin rock winder system.

package known as Megaflex ${ }^{5}$ of which the time intervals and energy costs are stated in Table 1. Although the shortest time interval in Table 1 is $1 \mathrm{~h}$, the demand costs are calculated over half hourly integration periods. Therefore the minimisation of the energy cost through near optimal hoist scheduling will also be done in half hourly periods thereby inherently minimising the network demand charge applicable during standard and peak periods. A cost vector is hence defined as $C=\left[\begin{array}{lllll}c_{0} & c_{1} & c_{2} & \ldots & c_{H-1}\end{array}\right]^{T}$ over a control horizon $H$ such that the elements in $C$ correspond to the values in Table 1 as indicated in Table 2 for the case of $D=1$ day or $H=48$.

The energy cost in period $k$ for the surface and underground winder therefore equates to $c_{k} u_{s}^{k} E_{s}$ and $c_{k} u_{g}^{k} E_{g}$ respectively. The summation of these two terms over the whole of $H$ results in the energy cost and linear objective function in (2) that is to be minimised:

$\min J=\min \left[\sum_{k=0}^{H-1} c_{k}\left(u_{s}^{k} E_{s}+u_{g}^{k} E_{g}\right)\right]$

\subsection{System constraints}

The objective function in (2) is subject to four constraints similar to the storage, production and process constraints found in [5-8]. The first constraint puts a limitation on the number of hoists that can be achieved by each winder during a 30 -min period. The hoist constraint is applicable to each period $k$ over $H$ and can be stated as $0 \leq u_{s}^{k} \leq 11$ for the surface winder and $0 \leq u_{g}^{k} \leq 17$ for the underground winder.

${ }^{5}$ Eskom, Eskom Retail Tariff Restructuring Plan, Non-local-authority Tariffs 2008/9, <http://www.eskom.co.za>. 
Table 1

Megaflex energy costs in c/kWh for 2008.

\begin{tabular}{|c|c|c|c|}
\hline Season/period & Peak $c_{p}$ & Standard $c_{s}$ & Off-peak $c_{o}$ \\
\hline Jan-MaySept-Dec & 20.52 & 12.77 & 9.10 \\
\hline Jun-Aug & 72.05 & 19.04 & 10.38 \\
\hline Time of day: $t \in$ & {$[7,10) \cup[18,20)$} & {$[6,7) \cup[10,18) \cup[20,22)$} & {$[0,6) \cup[22,24)$} \\
\hline
\end{tabular}

Table 2

Assigned values for the discrete cost function $C$.

\begin{tabular}{|c|c|c|c|c|c|c|c|}
\hline$t \in$ & {$[0,6)$} & {$[6,7)$} & {$[7,10)$} & {$[10,18)$} & {$[18,20)$} & {$[20,22)$} & {$[22,24)$} \\
\hline$c_{k}$ & $c_{0}, \ldots, c_{11}=c_{o}$ & $c_{12}, c_{13}=c_{s}$ & $c_{14}, \ldots, c_{19}=c_{p}$ & $c_{20}, \ldots, c_{35}=c_{s}$ & $c_{36}, \ldots, c_{39}=c_{p}$ & $c_{40}, \ldots, c_{43}=c_{s}$ & $c_{44}, \ldots, c_{47}=c_{o}$ \\
\hline
\end{tabular}

The second constraint is with respect to the upper (maximum) and lower (minimum) boundaries for the change-over and orepass system ore levels as indicated in Fig. 2. The level inequality constraints can be formulated as $m_{1 \min } \leq m_{1}^{k} \leq m_{1 \max }$ and $m_{2 \min } \leq$ $m_{2}^{k} \leq m_{2 \max }$.

A third constraint puts a lower boundary on the tons to be hoisted over the control horizon and is formulated in (3) as a horizon target constraint.

$\sum_{k=0}^{48 D-1} u_{s}^{k} R_{s} \geq D \times M_{\min } \quad$ for $k=0,1,2, \ldots, H-1$

Finally the fourth constraint takes into account mandatory routine daily winder maintenance or tests to be carried out on all winders during which time no hoisting of rock is allowed. For the surface winder it can be formulated as $u_{s}^{48 d+j}=0$ for $j=T_{S S}, T_{S S}+1$, $\ldots, T_{s s}+T_{m s}-1$ and $d=0,1, \ldots, D$. An equivalent formulation can be written for the underground winder in terms of $u_{g}, T_{s g}$ and $T_{m g}$.

\subsection{Discrete dynamic equation}

Underlying the winder system model is a discrete time dynamic system based on the basic dynamic programming model defined in [20]. This dynamic model is applied to the winder system's two state variables, $m_{1}$ and $m_{2}$, of which the result is formulated in the two discrete dynamic equations in (4).

$m_{1}^{k+1}=m_{1}^{k}+R_{g} u_{g}^{k}-R_{s} u_{s}^{k}$
$m_{2}^{k+1}=m_{2}^{k}-R_{g} u_{g}^{k}+m_{i n}^{k}$$\quad$ for $k=0,1,2, \ldots, H-1$

\section{Linear integer MPC formulation}

The above minimisation problem can be solved using linear programming after formulating the problem as a linear programming model (LPM). This formulation will require the objective function, cost vector and constraints to be explicitly stated at the beginning of each sampling instant $k$ within $H$. As presented in [8] the problem can be written in the form of formulations (5a) through (5d).

$\min _{u} f^{T} \cdot u$

$l b \leq u \leq u b$

$A \cdot u \leq b$

Aeq $\cdot u=b e q$

The product of the coefficient vector $f$ and the optimal real solution vector $u$ forms the objective energy cost function in (2) that is to be minimised. The two vectors $l b$ and $u b$ respectively represents the lower and upper hoist constraint boundaries for $u \cdot A$ and Aeq are matrices representing respectively the inequality and equality constraint coefficients along with vectors $b$ and beq containing real values thus forming the ore level and target constraints.
The linear programming formulation of (5) is combined with the MPC methodology presented in $[21,22]$ referred to as dynamic matrix control. The objective of the MPC control calculations over $H$ is to determine a sequence of manipulated inputs $u,\{u(k+j-1)$, $j=1,2, \ldots, H\}$, such that a set of predicted outputs over $P,\{\hat{y}(k+$ $j), j=1,2, \ldots, P\}$, reaches a target in an optimal manner [22]. The nature of this particular problem requires $P$ to equal $H$. Control calculations are based on multiple $j$-step ahead predictions of future outputs $\hat{y}(k+j)$, current measurements including actual outputs $y$, and on optimizing the objective function within a constant model horizon of $2 \mathrm{H} \leq \mathrm{N} \leq 3 \mathrm{H}$. The model horizon includes the effect of past, current and future control and uncontrolled actions [22]. Defining the problem in terms of the MPC methodology in $[21,22]$ results in the following reformulations.

The dynamic state formulation in (5) is reformulated in (6):

$$
\begin{aligned}
& \hat{m}_{1}^{k+j}=m_{1}{ }^{k}+\sum_{i=1}^{j}\left(R_{g} u_{g}{ }^{k+j-i}-R_{s} u_{s}^{k+j-i}\right) \\
& \hat{m}_{2}^{k+j}=m_{2}^{k}-\sum_{i=1}^{j} R_{g} u_{g}^{k+j-i}+\sum_{i=1}^{j} m_{i n}^{k+j-i}
\end{aligned}
$$

The objective function in (2) separated into the vectors $f$ and $u$ in (5a) is formulated in Eqs. (7) and (8) respectively:

$$
\begin{aligned}
f^{T}=\left[\begin{array}{cccc}
c_{k^{\prime}+j-1} E_{S} & c_{k^{\prime}+j-1} E_{g} & c_{k^{\prime}+j} E_{S} & c_{k^{\prime}+j} E_{g} \\
c_{k^{\prime}+j+1} E_{S} & c_{k^{\prime}+j+1} E_{g} \ldots & c_{k^{\prime}+j+H-1} E_{S} & c_{k^{\prime}+j+H-1} E_{g}
\end{array}\right] \\
\text { for } j=1,2,3, \ldots, P
\end{aligned}
$$

$u=\left[\begin{array}{lllllllll}u_{1} & u_{2} & u_{3} & u_{4} & u_{5} & u_{5} & \ldots & u_{(2 H-1)} & u_{2 H}\end{array}\right]$

$$
=\left[\begin{array}{llllllll}
u_{s}^{k} & u_{g}^{k} & u_{s}^{k+1} & u_{g}^{k+1} & u_{s}^{k+2} & u_{g}^{k+2} & u_{s}^{k+H-1} & u_{g}^{k+H-1}
\end{array}\right]
$$

where $k^{\prime}=k-48\left\lfloor\frac{k}{48}\right\rfloor=k-48 k_{t r}$ for $k=0,1,2, \ldots$ and \lfloor\rfloor denotes rounding down to the nearest integer.

Eq. (7) requires the cost vector $C$ to be reformulated to allow $N$ to move the required $(H-1)$ elements into the future. The reformulation of $C$ is given in (9) based on the values and notation of Tables 1 and 2.

$$
\begin{aligned}
& C=\left[\begin{array}{lllll}
c_{1}^{\prime} & c_{2}^{\prime} & c_{3}^{\prime} & \cdots & c_{2 D}^{\prime}
\end{array}\right]^{T} \\
& c_{n}^{\prime}=\left[\begin{array}{lllll}
c_{0} & c_{1} & c_{2} & \cdots & c_{47}
\end{array}\right]^{T}
\end{aligned}
$$

The first constraint, the hoist limits, can be written in the form of inequality ( $5 b$ ) such that $u b$ is defined as in (10) and that $l b$ contains $2 \mathrm{H}$ zero elements.

$$
\begin{aligned}
u b & =\left[\begin{array}{lllllll}
u b_{1} & u b_{2} & u b_{3} & u b_{4} & \cdots & u b_{(2 H-1)} & u b_{2 H}
\end{array}\right]^{T} \\
& =\left[\begin{array}{lllllll}
u b_{s} & u b_{g} & u b_{s} & u b_{g} & \cdots & u b_{s} & u b_{g}
\end{array}\right]^{T} \\
& =\left[\begin{array}{lllllll}
11 & 17 & 11 & 17 & \cdots & 11 & 17
\end{array}\right]^{T}
\end{aligned}
$$

The second constraint, the level inequality boundaries, can be formulated as in inequality $(5 \mathrm{c})$, which when written in the form 
presented in [22] results in the four inequalities given in (11) for the change over and orepass system levels.

$$
\begin{aligned}
& \sum_{i=1}^{j}\left(R_{g} u_{g}^{k+j-i}-R_{s} u_{s}^{k+j-i}\right) \leq\left(m_{1 \max }-m_{1}^{k}\right) \\
& \sum_{i=1}^{j}\left(-R_{g} u_{g}^{k+j-i}+R_{s} u_{s}^{k+j-i}\right) \leq\left(-m_{1 \min }+m_{1}^{k}\right) \\
& \sum_{\substack{j=1 \\
j}}^{j}\left(-R_{g} u_{g}^{k+j-i}\right) \leq\left(m_{2 \max }-m_{2}^{k}-\sum_{i=1}^{j} m_{i n}^{k+j-i}\right) \\
& \sum_{i=1}^{j} R_{g} u_{g}^{k+j-i} \leq\left(-m_{2 \min }+m_{2}^{k}+\sum_{i=1}^{j} m_{i n}^{k+j-i}\right) \\
& \text { for } j=1,2,3, \ldots, P
\end{aligned}
$$

Writing the horizon target constraint in inequality (3) in the form presented in [22] over a model horizon of $N$ periods results in:

$$
\begin{aligned}
& -\sum_{i=1}^{j} R_{s} u_{s}{ }^{k+j-i} \leq\left(\sum_{i=j+1}^{N} R_{s} u_{s}{ }^{k+j-i}-\frac{N}{48} M_{\min }\right) \\
& \quad \text { for } j=1,2,3, \ldots, P
\end{aligned}
$$

The formulation in (12) can be read as follows: "The tons to be hoisted during the next $j$ period(s) must be equal to or greater than the difference between the actual tons already hoisted to surface during the past $(N-j)$ periods and the product between the number of days within $N,(N / 48)$, and $M_{\text {min. }}$. From the summation on the right of the inequality in (12) it is clear that $N-1$ known past control actions are required. For simulation purposes (12) has to be implemented in phases until $k \geq N-1$ in order to create this history of known past control actions.

Finally the mandatory maintenance equality constraints for the underground winder can be written in the form of (5d) in (13a) through (13c) as follows:

$$
\begin{aligned}
& \sum_{d=0}^{D-1}\left(\sum_{i=T_{s g}}^{T_{s g}+T_{m g}-1} u_{g}^{i+48\left(d+k_{t r}\right)}\right)=0 \text { for } 0 \leq k^{\prime}<T_{s g} \\
& \sum_{d=0}^{D-1}\left(\sum_{i=k^{\prime}}^{T_{s g}+T_{m g}-1} u_{g}^{i+48\left(d+k_{t r}\right)}+\sum_{i=T_{s g}}^{k^{\prime}-1} u_{g}^{i+48\left(d+k_{t r}+1\right)}\right) \\
& \quad=0 \text { for } T_{s g} \leq k^{\prime}<T_{s g}+T_{m g}
\end{aligned}
$$

$$
\sum_{d=0}^{D-1}\left(\sum_{i=T_{s g}}^{T_{s g}+T_{m g}-1} u_{g}^{i+48\left(d+k_{t r}+1\right)}\right)=0 \text { for } T_{s g}+T_{m g} \leq k<48
$$

An equivalent formulation can be written for the surface winder in terms of $u_{s}, T_{s s}$ and $T_{m s}$.

\section{MPC integer solution algorithm}

The Branch and Bound (BnB) methodology described in [23] is adapted and applied as explained partially in [18] within an MPC algorithm to obtain a near optimal mixed integer hoist schedule solution to the LPM formulated in Section 4. Integer values are required because the winders cannot be controlled to complete a fraction of a hoist cycle within a 30-min period, but only complete cycles. A summary of the complete MPC algorithm including the adapted $\mathrm{BnB}$ methodology is provided below:

Step 1: Set $k=0$ and define the constants and initial conditions listed below where $m_{x}^{0}$ refers to the respective ore levels at the start of the simulation period.

$$
\begin{array}{llllllllll}
\text { Es } & \text { Eg } & M_{\text {blast }} & m_{1}^{0} & m_{1 \max } & m_{1 \min } & N & T_{s s} & T_{s g} \\
R_{s} & R_{g} & M_{\min } & m_{2}^{0} & m_{2 \max } & m_{2 \min } & D & T_{m s} & T_{m g}
\end{array}
$$

Step 2: Define $f$ and $u$ as in (7) and (8) and construct $l b, u b, A, b$, Aeq and beq for all $j=1,2,3, \ldots, P$.

Step 3: Minimise $f^{T} \cdot u$ subject to $l b \leq u \leq u b, A \cdot u \leq b$ and Aeq $\cdot u=b e q$ in order to obtain an optimal real solution for $u$ of (8).

Step 4: Using the first two elements $u_{1}$ and $u_{2}$ from the feasible optimal solution obtained for $u$, branch into four sub problems as illustrated in Fig. 3. Again $L\rfloor$ denotes rounding down and \lceil\rceil rounding up to the nearest integer.

For each sub problem create and add two equality constraints to the Aeq matrix and beq vector Aeq $\cdot u=b e q$ as defined in (14) using sub problem $c$ as an example:

$$
\left[\begin{array}{ll}
1 & 0 \\
0 & 1
\end{array}\right] \cdot\left[\begin{array}{l}
u_{1} \\
u_{2}
\end{array}\right]=\left[\begin{array}{l}
u_{s}^{k} \\
u_{g}^{k}
\end{array}\right]=\left[\begin{array}{l}
\left\lfloor u_{1}\right\rfloor \\
\left\lceil u_{2}\right\rceil
\end{array}\right]
$$

Step 5: Re-minimise $f^{T} \cdot u$ for each of the four sub problems with their specific added equality constraints now included into Aeq $\cdot u=$ beq.

Step 6: Select the values of $u_{s}^{k}$ and $u_{g}^{k}$ in the feasible sub problem having the lowest objective value that is not less than the optimal objective value obtained from the first minimisation in Step 3. Implement only these two values in period $k$.

Step 7: At the end of period $k$, record the actual number of hoists achieved by the surface winder $\hat{u}_{s}^{k}$ and the underground winder $\hat{u}_{g}^{k}$, as well as the actual feed-in rate $\hat{m}_{i n}^{k}$, during period $k$. As mentioned earlier the actual feed-in rate will be replaced by an historical average estimation for the purposes of this study.

Step 8: From the recorded values, update the historical data vector for $u_{s}$ required in (12) and calculate the new initial system state values for period $k+1$ :

$m_{1}^{k+1}=m_{1}^{k}+R_{g} \hat{u}_{g}^{k}-R_{s} \hat{u}_{s}^{k}$
$m_{2}^{k+1}=m_{2}^{k}-R_{g} \hat{u}_{g}^{k}+\hat{m}_{i n}^{k}$

Finally increment $k$ and repeat from Step 2.

It should be noted that the above algorithm differs quite substantially from commercially available solvers. If for example the control horizon is set for 2 days it results in 192 variables in $H$ to be solved at the beginning of each period. However, whereas the conventional solvers would actually endeavour to obtain an integer solution for each of the 192 variables, the algorithm presented only branches on the first two variables in $u$ irrespective of the magnitude of $H$ as these will be the only two variables to be implemented in accordance to the MPC algorithm. This adapted BnB algorithm therefore drastically reduces the computational effort and time required for the conventional $\mathrm{BnB}$ solvers by having to solve for only 2 variables instead of 192 in the case of $H$ being set to cover 2 days.

\section{Simulation study and discussion}

The study included various simulations investigating the impact of various factors on the hoist scheduling of which only a few will be summarised in this paper. By comparing the hoist schedules and related energy costs, the results presented in this paper will point out: 


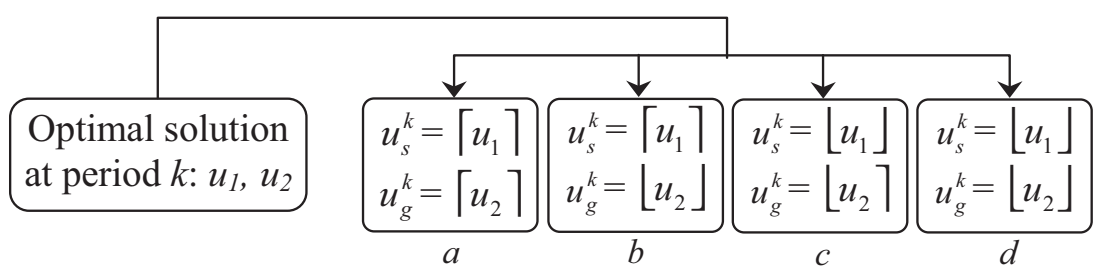

Fig. 3. Branching of the optimal solution at period $k$ into four subproblems.

Table 3

Initial state conditions and constraint values.

\begin{tabular}{llll}
\hline$E_{s}=130.85 \mathrm{kWh}$ & $R_{s}=23.5$ tons & $m_{1 \max }=5 \mathrm{ktons}$ & $T_{s s}=7$ \\
$E_{g}=42.91 \mathrm{kWh}$ & $R_{g}=13.5$ tons & $m_{1 \min }=500 \mathrm{ktons}$ & $T_{m s}=8$ \\
$N=2 H=96 D$ & $M_{\min }=8013.5$ tons & $m_{2 \max }=20 \mathrm{ktons}$ & $T_{s g}=8$ \\
$D=2$ & $M_{\text {blast }}=8200$ tons & $m_{2 \min }=7 \mathrm{ktons}$ & $T_{m g}=4$
\end{tabular}

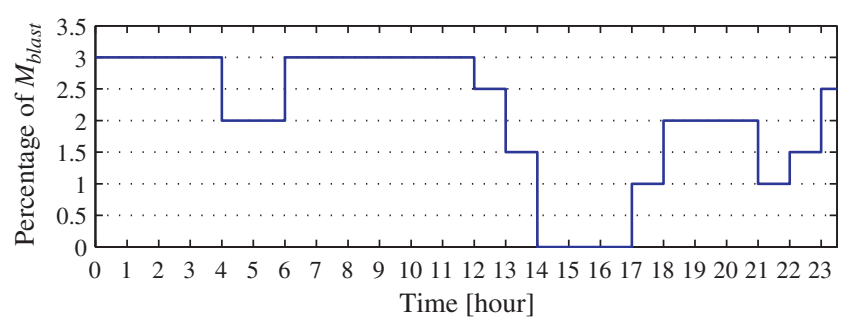

Fig. 4. Percentage function for $m_{i n}$ as a function of $M_{\text {blast }}$.

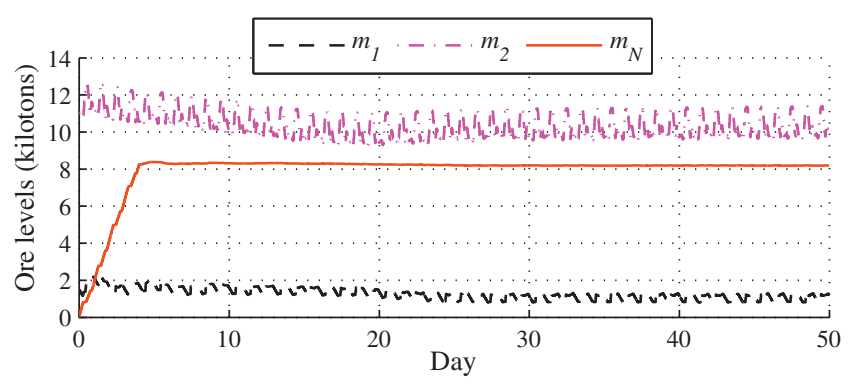

Fig. 5. Ore levels and transient response to obtain steady state values.

the near optimality of the adapted BnB method by applying and not applying the adapted BnB methodology,

the response of the controller to a TOU versus a flat rate tariff, the impact of introducing unplanned operational delays into the winder system.

Table 3 contains the values for the various constraints and initial conditions applied in the simulations below.

Fig. 4 shows the periodic percentage function for the feed-in rate of rock from the reefs into the orepass system $m_{i n}^{k}$ discussed in Section 3.1. The actual tons fed into the orepass system for each hour of the day is therefore estimated as the product of $M_{\text {blast }}$ and the percentage at that time according to Fig. 4.

\subsection{Obtaining steady state and historical hoist record values}

Fig. 5 shows the results of an optimal solution obtained after running a single simulation for 50 days. This simulation was done for two reasons. First to create the historical data record of $N-1$ known past control actions required in (12) and denoted as $u_{s m}$. Secondly to determine the initial steady state conditions for $m_{1}^{0}$ and $m_{2}^{0}$ to be used in further simulations. The values for $m_{1}^{0}$ and $m_{2}^{0}$

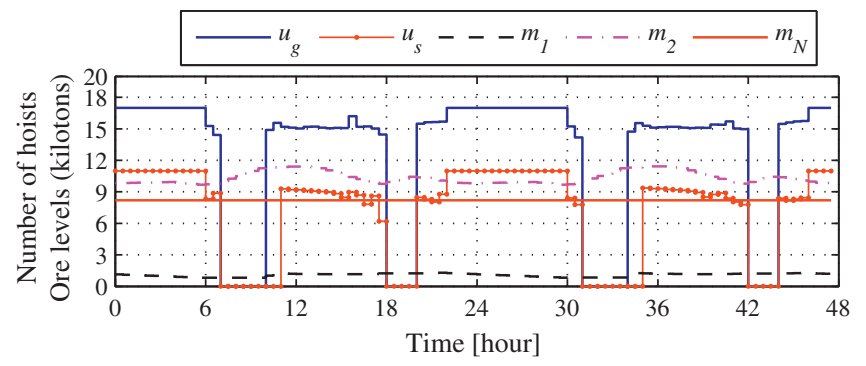

Fig. 6. An optimal hoist schedule with MPC applied and history taken into account.

were set to 1500 tons and 11000 tons respectively at the start of the 50 day simulation.

The top broken line in Fig. 5 represents the ore-pass system ore level $m_{2}$ and the bottom broken line the change-over ore level $m_{1}$. The solid line represents $m_{N}$, which is defined as the average tons hoisted per $24 \mathrm{~h}$ over all model horizon windows $N$ within the simulated period in accordance with (12). This running average $m_{N}$ is calculated at the end of each and every period using (15) and is used as a measure as to how well the controller adheres to the hoist target constraint.

$m_{N}^{k}=\frac{R_{s}}{N / 48} \sum_{i=k-(N-1)}^{k} u_{s}^{i}$

In steady state $m_{N}$ has an average of 8194 tons, which is noticeably closer to $M_{\text {blast }}$ than $M_{\min }$. This is to be expected in view of the fact that what is blasted underground needs to be hoisted to surface to prevent the ore transport system from saturating. The steady state values for $m_{1}$ and $m_{2}$ in Fig. 5 are 1168 tons and 9806 tons respectively and taken as the initial values of $m_{1}^{0}$ and $m_{2}^{0}$ for future simulations. Though the actual schedule of $u_{s}$ is not shown in Fig. 5 to avoid a cluttered graph, the last $N-1$ values were taken for constructing the historical hoist record $u_{s m}$.

\subsection{Near optimality of the adapted BnB method}

Control is now continued in time for a further two days from where Fig. 5 ended without introducing delays. Excluding the adapted BnB methodology in Step 4, 5 and 6 in the algorithm of Section 5 results in the optimal non-integer hoist schedule and ore levels in Fig. 6. Including the adapted BnB methodology to the same scenario results in the near optimal schedule in Fig. 7, which clearly is very similar to that of the optimal solution in Fig. 6 . The solid line remaining constant at approximately 8200 tons indicates the $m_{N}$ average as in Fig. 5. The top solid line without markers represents the underground winder schedule $u_{g}$ indicating the number of hoists to complete for each half-hourly period. Similarly the solid line with markers represents the surface winder schedule $u_{s}$. The broken lines are those representing $m_{1}$ and $m_{2}$ as in Fig. 5.

From the two figures above it is evident that no hoisting was scheduled during the expensive peak tariff periods. This happens provided that the hoist target or delays are not too high. Also, lim- 
Table 4

Numerical results and comparison for optimal, near optimal integer, flat rate and delay impact schedules.

\begin{tabular}{|c|c|c|c|c|}
\hline Solution: & Optimal (Fig. 6) & Near optimal (Fig. 7) & Flat Rate (Fig. 8) & Random delays (Fig. 9) \\
\hline$m_{N}$ at the end of day 2 [tons] & 8193.20 & 8212.70 & 8209.10 & 8013.50 \\
\hline Average 2-day energy cost & R 10664 & R 10727 & R 15504 & R 12254 \\
\hline
\end{tabular}

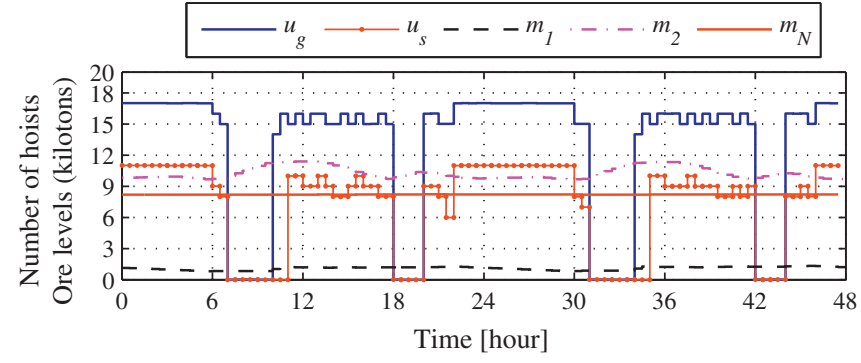

Fig. 7. A near optimal integer hoist schedule with the adapted BnB applied.

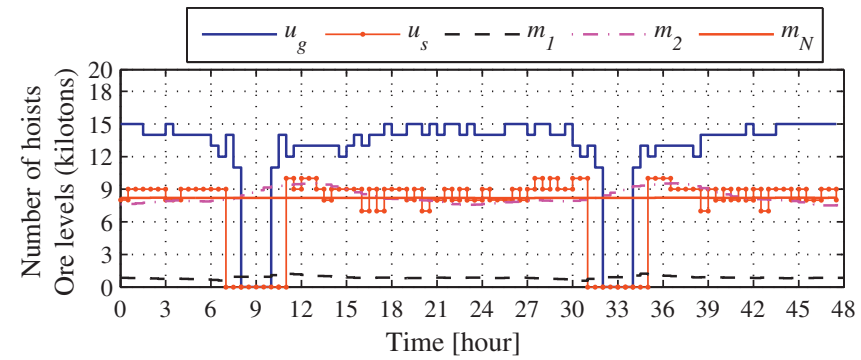

Fig. 8. A near optimal hoist schedule based on a weighted average flat rate tariff.

ited hoisting was scheduled during standard periods and maximum hoisting during the low cost off peak periods.

The near optimality of the adapted $\mathrm{BnB}$ solution is also supported by a numerical comparison of the results obtained for Figs. 6 and 7 in Table 4. A negligible difference is noted between the $m_{N}$ averages and the objectives or energy costs for the two solutions. Note also that the energy cost of the near optimal integer solution is slightly higher than the optimal solution.

\subsection{TOU versus flat rate tariff}

The comparative study in this scenario shows what the energy cost based on the TOU active energy cost in Table 1 would be if the scheduling of both winders were done based on a flat rate tariff thereby essentially ignoring the TOU tariff structure. The schedule based on a flat rate tariff in Fig. 8 shows that hoisting was scheduled and distributed almost evenly across all periods of the day except during the mandatory maintenance and testing times.

A comparison of the numerical results for Fig. 8 to that of Fig. 7 in Table 4 shows a mere 3.6 ton reduction in the $m_{N}$ average. However, applying the flat rate schedule on the TOU tariff resulted in an average 2-day energy cost increase of $44.5 \%$ from R 10727 to $R$ 15504 . It can also be stated that scheduling the hoists according to the TOU tariff results in an energy cost saving of ( $R$ 15504-R 10727 )/R $15504=30.8 \%$.

\subsection{Delay impact}

In order to illustrate the impact of delays on the winder control system, approximately 145 min delay per day were randomly enforced on the surface winder and $190 \mathrm{~min}$ on the underground winder. These delays were applied to the same conditions in Fig. 7,

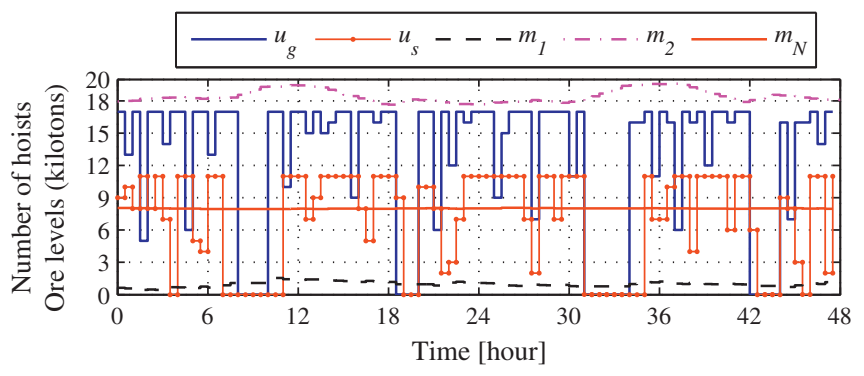

Fig. 9. A near optimal integer schedule with random delays enforced.

which resulted in the actual achieved hoisted schedule shown in Fig. 9.

Four significant differences are noted when comparing Fig. 9 with Fig. 7. First to note are the surface winder hoists scheduled during both evening and peak periods and the underground winder hoists during the morning peak period of the first day. Second to note is the increase in hoists scheduled on both days for both winders during standard periods. Thirdly the orepass system level is controlled at just below its upper limit of $20 \mathrm{ktons}$ and finally the $m_{N}$ average being controlled around the target of 8013.5 tons instead of above it at approximately $M_{\text {blast }}$. Simulations over longer periods showed that in time the change-over will also reach its upper boundary at $5 \mathrm{ktons}$ at which time the $m_{N}$ average will increase again to approximately $M_{\text {blast }}$.

Though not visible in Fig. 9, the $m_{N}$ average does drop below $M_{\text {min }}$ and $m_{1}$ below $m_{1 \text { min }}$ from time to time due to the unpredictability of the delays. The controller however continues to provide a sustainable hoist schedule by controlling the required levels around the boundaries. Therefore rather than seeing the boundaries as limits, they can be regarded as control set points.

The effectiveness of the MPC controller is revealed in the numerical results of Table 4 in that the $m_{N}$ average is controlled very close to $M_{\min }$ while keeping the average 2-day energy cost increase to just over R 1500 from the R 10727 for Fig. 7 to the R 12254 for Fig. 9. This is quite significant in view of the fact that almost $3 \mathrm{~h}$ of delay was enforced on each of the winders.

\section{Conclusion}

This paper showed the development of a constrained MILP dynamic optimisation problem of a twin rock winder system in order to obtain a near optimal hoist schedule by achieving a set hoist target at the lowest possible energy cost under unstable and unpredictable operating conditions. The problem was solved through an MPC algorithm using an adapted BnB methodology to find a near optimal mixed integer solution to the hoist scheduling problem at the start of each control period. The near optimal integer solutions were shown to be very close to the optimal non-integer solutions. The effectiveness of the MPC algorithm was illustrated by comparing the impact of using a TOU versus flat rate tariff as well as the inclusion of random unplanned or unscheduled delays into the winder system. Application of the MPC algorithm provided a $30 \%$ reduction in energy costs when applied on a TOU tariff compared to a flat rate tariff schedule. Despite almost $3 \mathrm{~h}$ of delays being enforced on both winders, the MPC algorithm managed to control 
G Model

EPSR-3213; No. of Pages 8

8

W. Badenhorst et al. / Electric Power Systems Research $x x x$ (2010) xxx-xxx

the tons hoisted around the daily target while keeping ore levels within their boundaries at a mere $14 \%$ increase in energy cost. Most importantly, the MPC algorithm maintains the ore levels such as to avoid the system from either running empty or saturating thereby ensuring that maximum hoisting can be done during off-peak periods while minimal hoisting is scheduled during the more expensive periods. This not only bares a financial advantage to the mine, but also aids in the improvement of the utilities load factor as part of its DSM objectives.

References

[1] H. Nilsson, The many faces of demand-side management, Power Eng. J. 8 (5) (1994) 207-210.

[2] S.J. Redford, The rationale for demand side management, Power Eng. J. 8 (5) (1994) 211-217.

[3] R. Pelzer, E.H. Mathews, D.F. le Roux, M. Kleingeld, A new approach to ensure successful implementation of sustainable demand side management (DSM) in South African mines, Energy 33 (2008) 1254-1263.

[4] G.J. Delport, Integrated electricity end-use planning in deep level mines, Doctonal thesis, University of Pretoria, 1994.

[5] S. Ashok, R. Banerjee, An optimisation mode for industrial load management, IEEE Trans. Power Syst. 16 (4) (2001) 879-884.

[6] S. Ashok, R.R. Banerjee, Optimal cool storage capacity for load management, Energy 28 (2) (2003) 115-126.

[7] S. Ashok, Peak-load management in steel plants, Apple. Energy 83 (5) (2006) 413-424.

[8] A. Middelberg, J. Chang, X. Kia, An optimal control model for load shifting - with application in the energy management of a colliery, Apple. Energy 86 (2009) 1266-1273.

[9] D.Q. Mayne, J.B. Rawlings, C.V. Roo, P.O.M. Scokaert, Constrained model predictive control: stability and optimality, Automatic 36 (6) (2000) 789-814
[10] G. de Nicolao, L. Mani, R. Scattolini, Stability and robustness of nonlinear reeding horizon control, in: F. Allgower, A. Zheng (Eds.), Nonlinear Model Predictive Control, Progress in Systems and Control Theory, Birkhauser Verlag, 2000, pp. 3-22.

[11] E.F. Camacho, C. Bordons, Model Predictive Control, 2nd ed., Springer, Berlin, 2004

[12] A.J. van Staden, J. Zhang, X. Kia, A model predictive control strategy for load shifting in a water pumping scheme with maximum demand charges, in: IEEE Power Tech Conf., Bucharest, Romania, 28 June - 2 July, 2009.

[13] X. Kia, J. Zhang, A. Elaiw, A model predictive control approach to dynamic economic dispatch problem, in: IEEE Power Tech Conf., Bucharest, Romania, 28 June - 2 July, 2009.

[14] A. Kusiak, L. Mingyang, Reheat optimization of the variable-air-volume box, Energy (2010), doi:10.1016/j.energy.2010.01.014.

[15] S.S. Voutetakis, P. Seferlis, S. Papadopoulou, Y. Kyriakos, Model-based control of temperature and energy requirements in a fluidised furnace reactor, Energy 31 (13) (2006) 2418-2427.

[16] K. Krüger, R. Franke, M. Rode, Optimization of boiler start-up using a nonlinear boiler model and hard constraints, Energy 29 (12-15) (2004) 2239-2251.

[17] M. Housing, A.N. Ajah, P.W. Heijnen, I. Bouwmans, P.M. Herder, Uncertainties in the design and operation of distributed energy resources: the case of microCHP systems, Energy 33 (10) (2008) 1518-1536.

[18] W. Badenhorst, J. Zhang, X. Kia, A near optimal hoist scheduling for deep level mine rock winders, in: IFAC Sym. Power Plants and Power Syst. Control, Tampere, Finland, 5-8 July, 2009.

[19] W. Badenhorst, J. Zhang, X. Kia, An MPC approach to deep level mine rock winder hoist control, in: IFAC Conf. Control Methodol. and Technol. for Energy Effic, Vilamoura, Portugal, 29-31 March, 2010.

[20] D.P. Bertsekas, Dynamic Programming and Optimal Control, Ind ed., Athena Scientific, Belmont, Massachusetts, 2001.

[21] C.E. Garcia, D.M. Prot, M. Morari, Model predictive control theory and practice - a survey, Automatic 25 (3) (1989) 335-348.

[22] D.E. Seborg, T.F. Edgar, D.A. Mellichamp, Process Dynamics and Control, end ed., John Wiley \& Sons, New York, 2006.

[23] G.L. Thompson, D.L. Wall, A branch and bound model for choosing optimal substation locations, IEEE Trans. Power Appear. Syst. 100 (5)(1981) 2683-2688.

Please cite this article in press as: W. Badenhorst, et al., Optimal hoist scheduling of a deep level mine twin rock winder system for demand side management, Electro. Power Syst. Res. (2011), doi:10.1016/j.epsr.2010.12.011 